\title{
Correlation Between Barrier Heights and Ideality Factors of Ni/n-Ge (100) Schottky Barrier Diodes
}

\author{
A. Chawanda* \\ Department of Physics, Midlands State University, Bag 9055 Gweru, Zimbabwe \\ J. M. Nel, F. D. Auret, W. Mtangi, C. Nyamhere, M. Diale ${ }^{\dagger}$ and L. Leach \\ Department of Physics, University of Pretoria, Pretoria, 002, South Africa
}

(Received 24 February 2010, in final form 31 March 2010)

\begin{abstract}
We computed the homogeneous Schottky barrier height (SBH) at ideality factor $(n)=1.0$ of Ni/nGe (100) Schottky diodes (SDs). The SDs were identically prepared by using resistive evaporation of $\mathrm{Ni}$ on $\mathrm{n}-\mathrm{Ge}(100)$. The SBHs and $n$ of these diodes (24 dots) were calculated from their experimental forward bias current-voltage $(I-V)$ and reverse bias capacitance-voltage $(C-V)$ measurements at room temperature. Even though the Schottky diodes were identically prepared, the values of the SBH from the $I-V$ characteristics varied from 0.487 to $0.508 \mathrm{eV}$, the ideality factor varied from 1.34 to 1.53 , and the SBH from the $C^{-2}-V$ characteristics varied from 0.358 to $0.418 \mathrm{eV}$. The Gaussian fits of the experimental SBH distributions obtained from the $C^{-2}-V$ and the $I-V$ characteristics yielded mean SBH values of $0.401 \pm 0.015$ and $0.503 \pm 0.006 \mathrm{eV}$, respectively. Furthermore, a homogeneous $\mathrm{SBH}$ value of approximately $0.535 \mathrm{eV}$ was also computed from an extrapolation of a linear plot of the experimental SBHs versus the ideality factors. The homogeneous SBHs, rather than the effective SBHs, of individual contacts or mean values should be used to discuss the theories and the physical mechanisms that determine the SBHs of SDs.
\end{abstract}

PACS numbers: 73.20.-r, 73.20.At, 73.40.Sx, 81.15.Ef

Keywords: Metal-semiconductor contact, Schottky barrier, Barrier inhomogeneity, Ideality DOI: $10.3938 /$ jkps.57.1970

\section{INTRODUCTION}

Metal-semiconductor (MS) interfaces are an essential part of virtually all semiconductor electronic and optoelectronic devices [1]. The physical properties of MS interfaces are widely studied, both for their basic physical properties and for their technological applications to electronic devices [2]. The MS structures are important research tools in the characterization of new semiconductor materials [3]. Their interface properties have a dominant influence on the performance, reliability and stability of devices $[1,4,5]$. A good MS contact is essential for the successful operation of electronic circuits and devices [6]. The electronic properties of the MS contacts are characterized by their barrier height (BH). The BH is the difference between the edge of the respective majority-carrier band of the semiconductor and the Fermi level at the interface [7]. Although Schottky barrier diodes (SBDs) have already been studied for more

*Corresponding Author; E-mail: albert.chawanda@up.ac.za; Tel: +27-12-420-3508; Fax: +27-12-362-5288

†Presenting Author; E-mail: mmantsae.diale@up.ac.za; Tel: +2712-420-4418; Fax: +27-12-362-5288 than fifty years, the fundamental mechanisms that determine the BH are still not fully understood [8-11]. It is only in the past decade that an inhomogeneous contact has been considered as an explanation for a voltagedependent BH [12-16]. Boyarbay et al. [17] suggested that the recent motivation for studying Schottky barrier formation is due to the recognition that both electronic and chemical equilibria have to be considered together across a reactive interface between a metal and a semiconductor, as surface states and metal-induced states fail to take into consideration the chemical equilibrium at the interface. The chemical equilibrium will result in an interfacial atomic rearrangement, interdiffusion, and inter-metallic compound formation, which should have a profound effect on the electronic equilibrium producing the Schottky barrier [18]. Therefore, the BH is likely to be a function of the interface atomic structure and the atomic inhomogeneities at MS interface, which are caused by grain boundaries, multiple phases, facets, defects, a mixture of different phases, etc. [19-22].

The measured values of the Schottky barrier height $(\mathrm{SBH})$ in the published literature are vastly scattered $[1,4,5,8]$. This may be due to the sensitivity of the Schottky interfaces to preparation procedures and to different 
techniques of measuring the $\mathrm{BH}$ [17]. In recent years, studies of SBH inhomogeneities have been carried out [16,19,23-30]. Freeouf et al. [23] reported on the influence of a small patch with a low $\mathrm{BH}$ within the contact area on the ideality factor and the essence of the contact area size in this effect. Song et al. [16] reported that the $\mathrm{BH}$ difference over the contact area was attributed to variations in the interfacial layer thickness and/or chemical composition and to the non-uniformity of the interfacial charges. Tung et al. [2,22,24,25] assumed lateral variations of the $\mathrm{BH}$ to model imperfect Schottky contacts. They depicted larger ideality factors and smaller effective BHs when they increased the inhomogeneity of the barriers. Some studies revealed that the experimentally observed dependences of the effective BHs and ideality factors of MS contacts could be explained by lateral inhomogeneities in the $\mathrm{BH}[27,30]$. The correlation between effective SBHs and ideality factors may be approximated by using a linear relationship [17].

Although studies have been performed to investigate the relationship between the effective $\mathrm{BHs}$ and the ideality factors of metal/Si diodes $[2,17,31]$, and the reactions of germanium (Ge) with nickel (Ni) [32-38], nothing has yet been reported on the relationship between effective $\mathrm{BHs}$ and ideality factors from forward bias currentvoltage $(I-V)$ and reverse bias capacitance-voltage $(C-V)$ characteristics of $\mathrm{Ni} / \mathrm{Ge}$ Schottky diodes. In most previous studies of $\mathrm{Ni} / \mathrm{Ge}$, interest has been focused on the formation and the morphology evolution of $\mathrm{Ni}$ on $\mathrm{n}-\mathrm{Ge}$ under rapid thermal annealing.

In this study, Ni Schottky diodes (24 diodes) were fabricated on n-Ge (100) under experimentally identical conditions. Our purpose was to experimentally investigate the relationship between the effective $\mathrm{BHs}$ and the ideality factors obtained from the forward bias $I$ $V$ and reverse bias $C-V$ characteristics of the $\mathrm{Ni} / \mathrm{n}-\mathrm{Ge}$ (100) Schottky diodes. The homogeneous BH value for Ni Schottky contacts was obtained from the linear relationship between the experimental effective BHs and ideality factors. The rest of the paper is organized as follows: In Sec. II, we briefly describe the experimental procedure. Results and a discussion will be presented in Sec. III. A summary of the work will be given in Sec. IV.

\section{EXPERIMENTAL PROCEDURE}

We used bulk-grown, (100)-oriented, n-type Ge, doped with antimony $(\mathrm{Sb})$ to a density of about $1.5-2 \times 10^{15}$ $\mathrm{cm}^{-3}$ and supplied by Umicore. Before metallization, the samples were first degreased and subsequently etched in a mixture of $\mathrm{H}_{2} \mathrm{O}_{2}(30 \%): \mathrm{H}_{2} \mathrm{O}$ (1:5) for 1 minute. Immediately after cleaning, the samples were inserted into a vacuum chamber where AuSb (0.6\%), 120-nm thick, was deposited by resistive evaporation on the back surfaces as Ohmic contacts. The samples were then annealed at 350 ${ }^{\circ} \mathrm{C}$ in $\mathrm{Ar}$ for 10 minutes to minimize the contact resistiv- ity of the Ohmic contacts [39]. Before Schottky contact deposition, the samples were again chemically cleaned as described above. Ni Schottky contacts were deposited onto Ge wafers by using vacuum resistive evaporation at a pressure of about $10^{-6}$ Torr. The contacts were $0.6 \mathrm{~mm}$ in diameter and 30-nm thick. The thickness of the metal layer and the deposition rates were monitored by using an INFICON XTC 751-001-G1 quartz crystal thickness monitor. After the contact fabrication, the SBDs were characterized by using $I-V$ and $C-V$ measurements at room temperature.

\section{RESULTS AND DISCUSSION}

The BHs of the contacts were deduced from the $I$ $V$ characteristics, which were analyzed by using the thermionic emission model given by the following equation $[5,9]$ :

$$
I(V)=I_{0} \exp \left(\frac{q V}{n k T}\right)\left[1-\exp \left(-\frac{q V}{k T}\right)\right],
$$

with

$$
I_{0}=A^{*} A T^{2} \exp \left(-\frac{q \Phi_{B}}{k T}\right),
$$

where $I_{0}$ is the saturation current derived from the straight line intercept of the $\ln (I)-V$ plot at $V=0, V$ is the forward bias voltage, $T$ is the absolute temperature, $q$ is the electronic charge, $k$ is the Boltzmann constant, $A$ is the effective diode area, and $A^{*}=4 \pi q m^{*} k^{2} / h^{3}$ is the effective Richardson constant, 50 Acm-2 K-2 for n-Ge [40], with $m^{*}$ being the effective mass for the electrons perpendicular to the layer plane, $m_{0}$ the rest mass of the electron, and $\Phi_{B}$ the zero bias effective SBH. From Eq. (2), we have:

$$
\Phi_{B}=\frac{k T}{q} \ln \left(\frac{A^{*} A T^{2}}{I_{0}}\right),
$$

and $n$ is the ideality factor, which can be obtained accurately from the slope of the linear part of an $\ln (I)$ versus $V$ plot, assuming pure thermionic emission can be obtained from Eq. (1) as

$$
n=\frac{q}{k T} \frac{d V}{d(\ln I)},
$$

which is equal to 1.0 for an ideal diode and usually has a value greater than unit.

The measured $I-V$ plots of the Ni/n-Ge (100) Schottky barrier diodes at room temperature are shown in Fig. 1. We performed least-squares fits of Eq. (1) to the linear part of the measured $I-V$ plots. From these fits, the experimental values of $I_{0}$ and $n$ were determined from the intercepts and the slopes of the forward bias $\ln (I)$ 


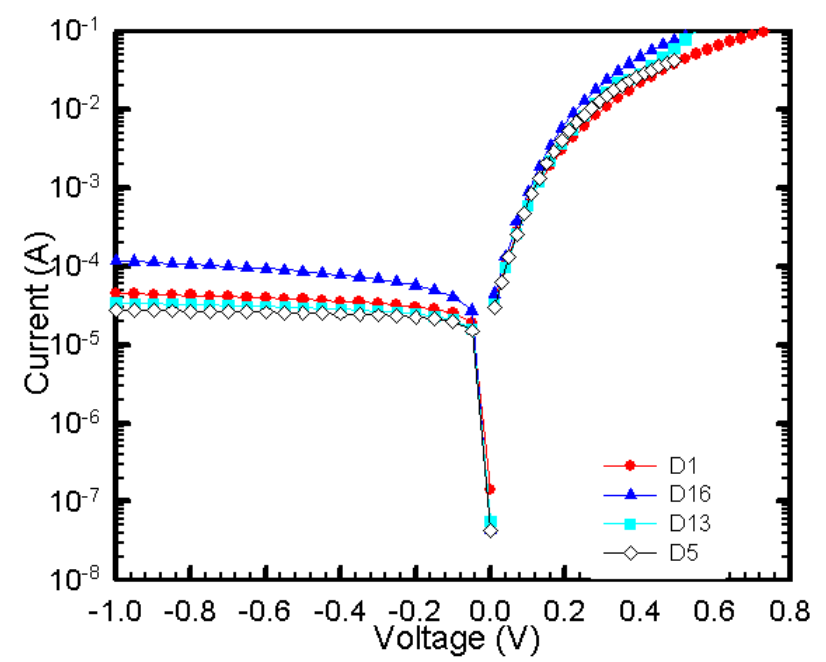

Fig. 1. (Color online) Plot of the forward and the reverse bias current-voltage $(I-V)$ characteristics for the four selected samples of Ni/n-Ge (100) Schottky diodes at room.

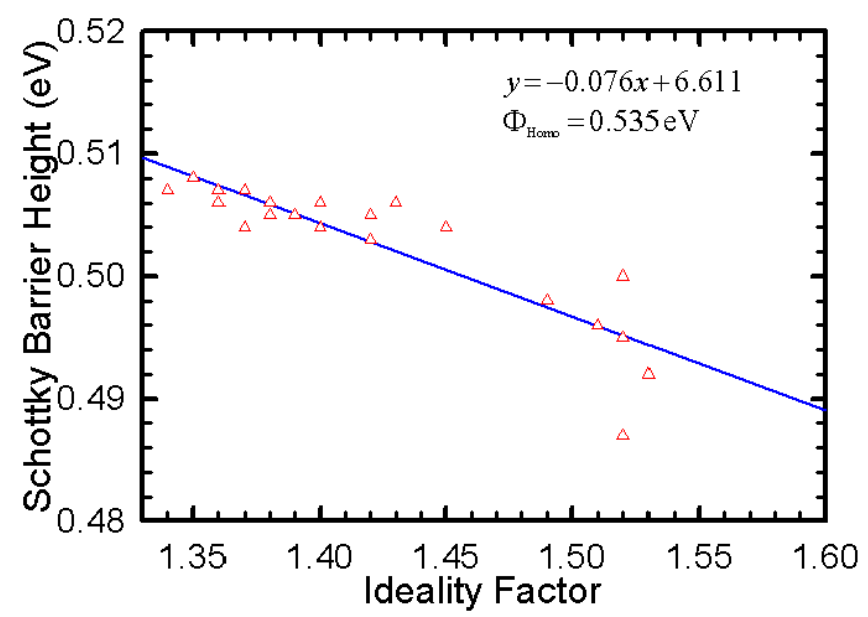

Fig. 2. Plot of the experimental barrier heights versus ideality factors of the Ni/n-Ge (100) Schottky barrier diodes at room temperature.

vs. $V$ plot at room temperature, respectively. Once $I_{0}$ is known, $\Phi_{B}$ can be computed from Eq. (3). The effective $\mathrm{BHs}$ for the diodes varied from 0.487 to $0.508 \mathrm{eV}$, and the ideality factors ranged from 1.34 to 1.53 , with standard deviations of $0.06 \mathrm{eV}$ and 0.064 , respectively. As can be seen from the data, the effective $\mathrm{BHs}$ and ideality factors from the $I-V$ characteristics varied from diode to diode even though were identically prepared on the same sample.

Figure 2 shows a plot of the effective BHs as a function of the respective ideality factors at room temperature. As can be seen from Fig. 2, there is a linear relationship between the experimental effective BHs and ideality factors of the Schottky structures. The BHs become smaller as the ideality factors increases. Güler et al. [31] also mentioned that higher ideality factors among identically prepared diodes were often found to accompany lower

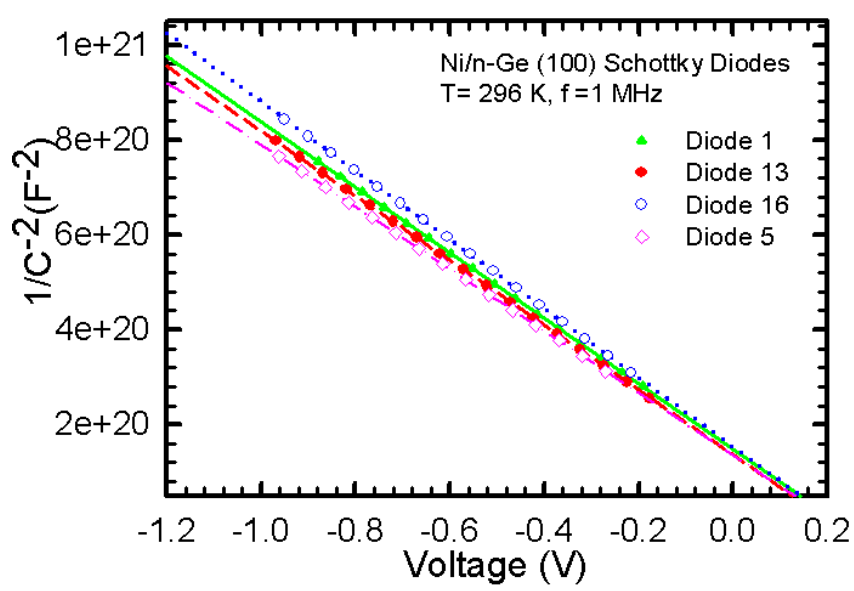

Fig. 3. (Color online) Reverse bias $C^{-2}-V$ characteristics for the four selected samples of Ni/n-Ge(100) Schottky structures at a frequency of $1 \mathrm{MHz}$ and at room temperature.

observed BHs. This may be attributed to lateral barrier inhomogeneities in SDs $[1,25,30,41,42]$. Such behaviors of the $\mathrm{BH}$ and the ideality factor can be explained by means of the bias dependence of the saddle-point potential of an inhomogeneous BH [1,25]. Mönch [4] also proposed that interface defects induced during contacts fabrication could exist, in addition to metal-induced gap states (MIGS), and alter the $\mathrm{BH}$. The defects give rise to additional discrete levels in the band gap, and the Fermi level is pinned to one of these levels, possibly quite far away from the charge neutrality level [2]. The straight line in Fig. 2 is the least-squares fit to the experimental data. A laterally homogeneous $\mathrm{BH}$ value of $0.535 \mathrm{eV}$ for the Ni/n-Ge (100) Schottky structures was obtained from the extrapolation of the plot to $n=1.0$. The homogeneous $\mathrm{BHs}$, rather than effective $\mathrm{BHs}$, of individual contacts or their mean values should be used to discuss theories on the physical mechanisms that determine the BHs of MS contacts $[27,43]$.

For a number of selected Ni/n-Ge (100) Schottky diodes, the reverse bias $C^{-2}-V$ characteristics at $1.0 \mathrm{MHz}$ are depicted in Fig. 3. The plots of as $C^{-2}$ a function of reverse bias voltage are linear, which indicates the formation of SDs [44] and a constant donor concentration. In Schottky diodes, the depletion layer capacitance $(C)$ can be expressed as $[5,9]$ :

$$
\frac{1}{C^{2}}=\frac{2\left(V_{0}-V\right)}{q \varepsilon_{s} A^{2} N_{D}},
$$

where $A$ is the area of the diode, is the permittivity of the semiconductor, is the carrier doping density, $V$ is the magnitude of the reverse bias, and is the intercept of with the voltage axis and is given by

$$
V_{0}=V_{D}-k T / q .
$$

The BH can be obtained from Fig. 3 as

$$
\Phi_{B}(C-V)=V_{D}+V_{n}-\Delta \Phi_{B},
$$




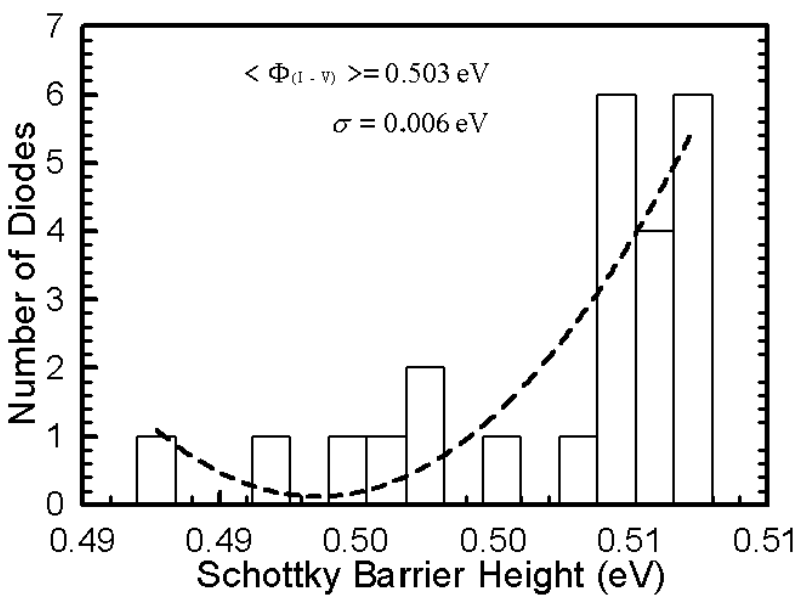

Fig. 4. Distribution of barrier heights from the forward bias $I-V$ characteristics of the Ni/n-Ge (100) Schottky structures at room temperature.

where $V_{n}$ is the energy difference between the bulk Fermi level and the conduction band edge, $V_{D}$ is the diffusion potential, and $\Delta \Phi_{B}$ is the image force barrier lowering and is given by $[5,9]$

$$
\Delta \Phi_{B}=\left[\frac{q E_{m}}{4 \pi \varepsilon_{s} \varepsilon_{0}}\right]^{1 / 2}
$$

with $E_{m}$ being the maximum electric field and being given by

$$
E_{m}=\left[\frac{2 q N_{D} V_{0}}{\varepsilon_{s} \varepsilon_{0}}\right]^{1 / 2}
$$

The capacitance-voltage $\mathrm{BH}$ values for the $\mathrm{Ni} / \mathrm{n}-\mathrm{Ge}(100)$ diodes ranged from 0.358 to $0.418 \mathrm{eV}$.

Figure 4 shows the statistical distribution of BHs from the forward bias $I-V$ plots of the Ni/n-Ge (100) Schottky barrier diodes (24 dots), and Fig. 5 shows the statistical distribution of BHs from the reverse bias $C^{-2}-V$ plots of the same diodes. The Gaussian distribution function was used to obtain fits to the histograms. The probability of SBH, $P\left(\Phi_{B}\right)$, has the form $[45,46]$ :

$$
P\left(\Phi_{B}\right) \frac{1}{\sigma \sqrt{2 \pi}} \exp \left\{-\frac{\left(\Phi_{B}-\bar{\Phi}_{B}\right)^{2}}{2 \sigma^{2}}\right\}
$$

where $\bar{\Phi}_{B}$ is the mean value of SBH, $\sigma$ is the standard deviation, and $1 / \sigma \sqrt{2 \pi}$ is the normalization constant.

The statistical analysis of the $I-V$ BHs reveals a mean $\mathrm{SBH}$ value of $0.503 \mathrm{eV}$ with a standard deviation of 0.006 $\mathrm{eV}$. In the distribution of BHs from the reverse bias $\mathrm{C}^{-2}$ $V$ characteristics at $1.0 \mathrm{MHz}$ (Fig. 5) and statistical analysis yielded a mean $\mathrm{BH}$ value of $0.401 \mathrm{eV}$ with a standard deviation of $0.015 \mathrm{eV}$. Due to the different nature of the measurement techniques, BHs deduced from $I-V$ and $C^{-2}-V$ are not always the same [31]. Although, in general, BHs from $C-V$ measurements are higher than

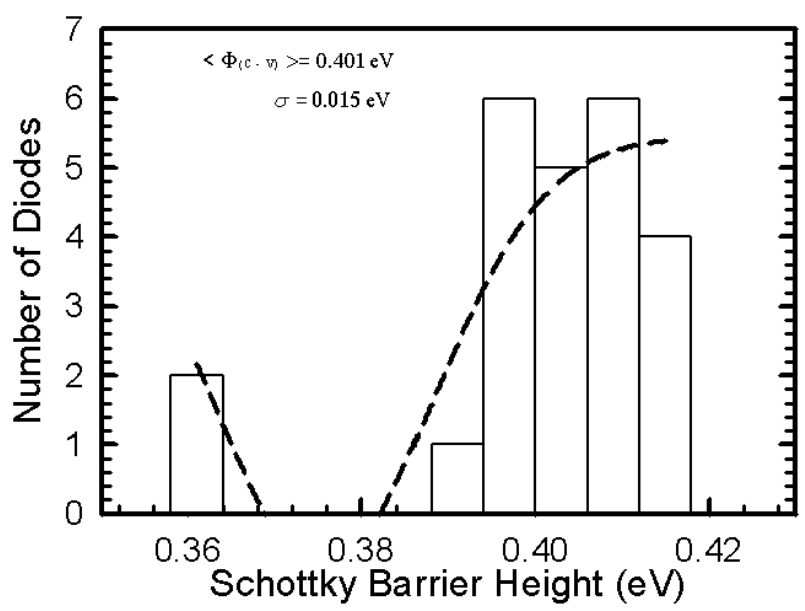

Fig. 5. Distribution of barrier heights from the reverse bias $C^{-2}-V$ characteristics of the Ni/n-Ge (100) Schottky structures at $1 \mathrm{MHz}$ and room temperature.

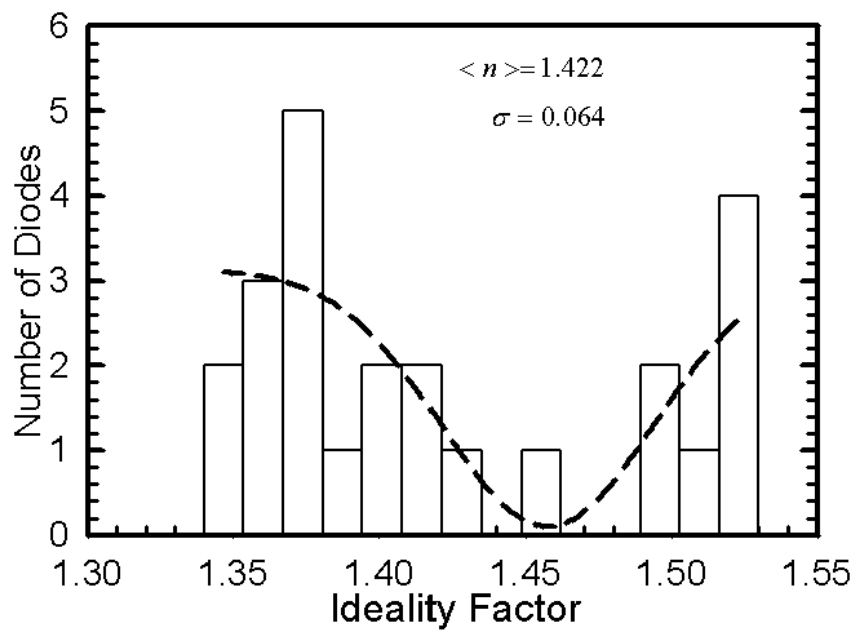

Fig. 6. Distribution of ideality factors from the forward bias $I-V$ characteristics of the $\mathrm{Ni} / \mathrm{n}-\mathrm{Ge}(100)$ diodes at room temperature.

BHs from $I-V$ measurements, in our study, we obtained $I-V$ BHs that were higher than $C-V$ BHs. Therefore, further studies are needed to clarify these results.

Figure 6 shows the statistical distribution of ideality factors from the forward bias $I-V$ characteristics. A Gaussian distribution function was used to obtain a fit to the histogram. The statistical analysis of the ideality factors yielded an average value of 1.422 with a standard deviation of 0.064. Our data clearly show that the diodes have ideality factors that are considerably larger than 1 , the value determined by the image-force effect only $[1,5,9,16,46]$. Therefore, these diodes are patchy $[4,27,30,47]$. Ideality factors greater than 1.0 indicate that the transport properties are not well modeled by thermionic emission alone although the contacts remain rectifying [48]. Explanations for the deviation of the ideality factor from unit ranged from assumptions of a 
generation-recombination current in the space-charge region $[5,8,17]$ to interface dielectric layers to field emission [17] to thermionic field emission [49] due to secondary mechanisms at the interface $[30,50]$. For example, interface defects may lead to a lateral inhomogeneous distribution of $\mathrm{BHs}$ at the interface, resulting in the presence of a wide distribution of low-SBH patches and in excess current leading to a deviation from ideal thermionic emission behavior at low voltages and temperatures.

\section{SUMMARY}

Ni Schottky diodes (24 dots) on n-Ge (100) were fabricated by using resistive deposition under experimentally identical conditions. The BHs and the ideality factors were obtained from the individual $I-V$ characteristics of the MS contacts. We showed that the BHs and the ideality factors varied from diode to diode even though they are were identically fabricated. A laterally homogeneous $\mathrm{BH}$ value of $0.535 \mathrm{eV}$ was obtained for the Schottky contacts from the linear relationship between the $I-V$ effective BHs and ideality factors. A statistical analysis yielded a mean effective $\mathrm{SBH}=0.503 \pm 0.006 \mathrm{eV}$ and a mean ideality factor $=1.422 \pm 0.064$ for these devices from the $I-V$ characteristics; a mean effective $\mathrm{SBH}=$ $0.401 \pm 0.015 \mathrm{eV}$ was obtained for these devices from the $C-V$ characteristics. Furthermore, experimental data for the $\mathrm{Ni} / \mathrm{n}-\mathrm{Ge}(100)$ contacts were interesting experimental illustration of the theoretical predictions [27].

\section{ACKNOWLEDGMENTS}

This work has been made possible by financial assistance from the South African National Research Foundation.

\section{REFERENCES}

[1] R. T. Tung, Mater. Sci. Eng., R 35, 1 (2001).

[2] M. Sağlam, F. E. Cimilli and A. Türüt, Physica B 348, 397 (2004)

[3] Ö. F. Yüksel, Physica B 404, 1993 (2009).

[4] W. Mönch, Semiconductor Surf. And Interfaces, 2nd ed. (Springer, Berlin 1995).

[5] M. Sze, Physics of Semiconductor Devices, 2nd ed. (Wiley, New York, 1981).

[6] A. R. Saha, S. Chattopadhyay and C. K. Maiti, Mater. Sci. Eng., B 114, 218 (2004).

[7] T. U. Kampen, W. Mönch, Surf. Sci. 331, 490 (1995).

[8] E. Hökelek and G. Y. Robinson, Solid-State Electron. 99, 24 (1981).

[9] E. H. Rhoderick and R. H. Williams, MetalSemiconductor Contacts (Clarendon Press, Oxford, 1988).
[10] G. A. Barti and M. Schlilter, Phys. Rev. B 33, 7346 (1986).

[11] S. Chand and S. Bala, Physica B 390, 179 (2007).

[12] S. Asubay, Ö. Güllü and A. Türüt, Appl. Surf. Sci. 254, 3558 (2008).

[13] L. Qiang and J. Wanqi, Semicond. Sci. Technol. 21, 72 (2006).

[14] S. H. Huang, Y. Tian and F. Lu, Appl. Surf. Sci. 234, $362(2004)$.

[15] E. Dobrocka and J. Osvald, Appl. Phys. Lett. 65, 575 (1994).

[16] Y. P. Song, R. L. van Meirhaeghe, W. H. Laflere and F. Cardon, Solid-State Electron. 29, 633 (1986).

[17] B. Boyarbay, H. Çetin, M. Kaya and E. Ayyildiz, Microelectron. Eng. 85, 721 (2008).

[18] R. D. Thomson and K. N. Tu, J. Appl. Phys. 53, 4285 (1982).

[19] J. H. Werner and H. H. Guttler, J. Appl. Phys. 69, 1522 (1991).

[20] S. Chand and J. Kumar, J. Appl. Phys. 82, 5005 (1997).

[21] H. Çetin and E. Ayyildiz, Semicond. Sci. Technol. 20, $625(2005)$

[22] R. T. Tung, Phys. Rev. B 45, 13509 (1992).

[23] J. L. Freeouf, T. N. Jackson, S. E. Laux and J. M. Woodall, Appl. Phys. Lett. 40, 634 (1982).

[24] R. T. Tung, A. F. J. Levi, J. P. Sullivan and F. Schrey, Phys. Rev. Lett. 66, 72 (1991).

[25] J. P. Sullivan, R. T. Tung, M. R. Pinto and W. R. Graham, J. Appl. Phys. 70, 7403 (1991).

[26] J. H. Werner and H. H. Güttler, J. Appl. Phys. 73, 1315 (1993).

[27] R. F. Schmitsdorf, T. U. Kampen and W. Mönch, J. Vac. Sci. Technol., B 15, 1221 (1997).

[28] K. Maeda, J. Vac. Sci. Technol., B 19, 268 (2001).

[29] L. E. Calvet, R. G. Wheeler and M. A. Reed, Appl. Phys. Lett. 80, 1761 (2002).

[30] W. Mönch, J. Vac. Sci. Technol., B 17, 1867 (1999).

[31] G. Güler, Ş. Karataş, Ö. Güllü and Ö. F. Bakkaloğlu, J. Alloys Compd. 486, 343 (2009).

[32] A. Thanailakis and D. C. Northrop, Solid-State Electron. 16, 1383 (1973)

[33] M. Wittmer, M.-A. Nicolet and J. W. Mayer, Thin Solid Films 42, 51 (1977).

[34] K. Y. Lee, S. L. Liew, S. J. Chua, D. Z. Chi, H. P. Sun and X. Q. Pan, Mater. Res. Soc. Symp. Proc. 810, C2.4.1 (2004).

[35] S. Zhu and A. Nakajima, Jpn. J. Appl. Phys. 44, L753 (2005).

[36] Q. Zhang, N. Wu, T. Osipowicz, L. K. Bera and C. Zhu, Jpn. J. Appl. Phys. 44, L1389 (2005).

[37] H. De-Dong, L. Xiao-Yan, K. Jin-Feng, X. Zhi-Liang, D. Gang and H. Ru-Qi, Chin. Phys. 14, 1041 (2005).

[38] D. Ikeno, Y. Kaneko, H. Kondo, M. Sakashita, A. Sakai, M. Ogawa and S. Zaima, Jpn. J. Appl. Phys. 46, 1865 (2007).

[39] Germanium Silicon: Physics and Materials, Semiconductors and Semi-metals, edited by R. Hull and J. C. Bean (Academic, San Diego, 1999), Vol. 56.

[40] H. B. Yao, C. C. Tan, S. L. Liew, C. T. Chua, C. K. Chua, R. Li, R. T. P. Lee, S. J. Lee and D. Z. Chi, in International Workshop on Junction Technology Proc (Shanghai, China, May 15-16, 2006), p. 164.

[41] T. U. Kampen and W. Mönch, Surf. Sci. 331, 490 (1995). 
[42] G. Güler, Ö. Güllü, Ö. F. Bakkaloglu and A. Türüt, Physica B 403, 221 (2008).

[43] H. Çetin, B. Şahin, E. Ayyilidiz and A. Türüt, Semicond. Sci. Technol. 19, 1113 (2004).

[44] V. Saxena and R. Prakash, Polym. Bull. 45, 267 (2000).

[45] H. H. Güttler and J. H. Werner, Appl. Lett. 56, 1113 (1990).

[46] J. H. Werner and H. H. Güttler, J. Appl. Phys. 69, 1522
(1991) .

[47] W. Mönch, Phys. Rev. B 37, 7129 (1988).

[48] H. Doğan, N. Yildirim and A. Turut, Microelectron. Eng. 85, 721 (2008) .

[49] E. Ayyildiz, H. Çetin and Z. Horváth, Appl. Surf. Sci. 252, 1153 (2005).

[50] S. Chand and S. Bala, Physica B 390, 179 (2007). 